\title{
Local convergence for an eighth order method for solving equations and systems of equations
}

https://doi.org/10.1515/nleng-2017-0105

Received August 27, 2017; revised December 6, 2017; accepted April 19, 2018.

Abstract: The aim of this study is to extend the applicability of an eighth convergence order method from the $k-$ dimensional Euclidean space to a Banach space setting. We use hypotheses only on the first derivative to show the local convergence of the method. Earlier studies use hypotheses up to the eighth derivative although only the first derivative and a divided difference of order one appear in the method. Moreover, we provide computable error bounds based on Lipschitz-type functions.

Keywords: Banach space, local convergence, convergence order, Lipschitz condition

MSC: 65H10, 65G99, 47H17, 49M15

\section{Introduction}

Let $F: \Omega \subseteq \mathcal{B}_{1} \longrightarrow \mathcal{B}_{2}$ be a differentiable operator in the sense of Fréchet between the Banach spaces $\mathcal{B}_{1}$ and $\mathcal{B}_{2}$ and $\Omega$ be a convex set. In this study, we consider the problem of approximating the solution $x^{\star}$ of nonlinear equation

$$
F(x)=0
$$

We consider the following eighth order method considered in [3] for approximating $x^{\star}$

$$
\begin{aligned}
y_{n} & =x_{n}-F^{\prime}\left(x_{n}\right)^{-1} F\left(x_{n}\right) \\
z_{n} & =y_{n}-5 F^{\prime}\left(x_{n}\right)^{-1} F\left(y_{n}\right) \\
w_{n} & =z_{n}+\frac{1}{5} F^{\prime}\left(x_{n}\right)^{-1}\left(16 F\left(y_{n}\right)-F\left(z_{n}\right)\right) \\
x_{n+1} & =w_{n}-Q\left(s_{n}\right) F^{\prime}\left(x_{n}\right)^{-1} F\left(w_{n}\right),
\end{aligned}
$$

where $x_{0} \in \Omega$ is an initial point, $s_{n}=I-$ $5 F^{\prime}\left(x_{n}\right)^{-1}\left[y_{n}, x_{n} ; F\right], s: \mathcal{B}_{1}^{2} \longrightarrow \mathcal{L}\left(\mathcal{B}_{1}, \mathcal{B}_{1}\right)$ and $Q$ :

\footnotetext{
Ioannis K. Argyros, Department of Mathematical Sciences, Cameron University, Lawton, OK 73505, USA, E-mail: iargyros@cameron.edu *Corresponding Author: Santhosh George, Department of Mathematical and Computational Sciences, NIT Karnataka, India-575 025, E-mail: sgeorge@nitk.ac.in
}

$\mathcal{L}\left(\mathcal{B}_{1}, \mathcal{B}_{2}\right) \longrightarrow \mathcal{L}\left(\mathcal{B}_{1}, \mathcal{B}_{1}\right)$, where $s\left(x_{n}, y_{n}\right)=s_{n}$. Finding solution for the equation (1.1) is an important problem in mathematics due to its wide applications. So improving the order of convergence of iterative method for solving (1.1) is also an important problem in mathematics. In [3] the existence of the Fréchet derivative of $F$ of order up to the eighth was used to show the eighth order of convergence of method (1.2) in the special case when $\mathcal{B}_{1}=\mathcal{B}_{2}=$ $\mathbb{R}^{k}$. This assumption on the higher order Fréchet derivatives of the operator $F$ restricts the applicability of method (1.2). For example consider the following:

Example 1.1. Let $\mathcal{B}_{1}=\mathcal{B}_{2}=C[0,1], \Omega=\bar{B}\left(x^{*}, 1\right)$ and consider the nonlinear integral equation of the mixed Hammerstein-type [1, 2, 6-9, 12] defined by

$$
x(s)=\int_{0}^{1} G(s, t)\left(x(t)^{3 / 2}+\frac{x(t)^{2}}{2}\right) d t
$$

where the kernel $G$ is the Green's function defined on the interval $[0,1] \times[0,1]$ by

$$
G(s, t)= \begin{cases}(1-s) t, & t \leq s \\ s(1-t), & s \leq t\end{cases}
$$

The solution $x^{\star}(s)=0$ is the same as the solution of equation (1.1), where $F: C[0,1] \longrightarrow C[0,1])$ is defined by

$$
F(x)(s)=x(s)-\int_{0}^{1} G(s, t)\left(x(t)^{3 / 2}+\frac{x(t)^{2}}{2}\right) d t .
$$

Notice that

$$
\left\|\int_{0}^{1} G(s, t) d t\right\| \leq \frac{1}{8} .
$$

Then, we have that

$$
F^{\prime}(x) y(s)=y(s)-\int_{0}^{1} G(s, t)\left(\frac{3}{2} x(t)^{1 / 2}+x(t)\right) d t
$$

so since $F^{\prime}\left(\chi^{\star}(s)\right)=I$,

$$
\left\|F^{\prime}\left(x^{\star}\right)^{-1}\left(F^{\prime}(x)-F^{\prime}(y)\right)\right\| \leq \frac{1}{8}\left(\frac{3}{2}\|x-y\|^{1 / 2}+\|x-y\|\right) .
$$


One can see that, higher order derivatives of $F$ do not exist in this example.

Our goal is to weaken the assumptions in [3] and apply the method for solving equation (1.1) in Banach spaces, so that the applicability of the method (1.2) can be extended using hypotheses only on the first derivative and the divided difference of order one which actually appear in method (1.2). We rely on COC and ACOC for the determination of the order of convergence. Our technique can be used on other iterative methods along the same lines [1-27].

The rest of the paper is organized as follows. In Section 2 we present the local convergence analysis. We also provide a radius of convergence, computable error bounds and a uniqueness result not provided in [3]. Special cases and numerical examples are given in the last section.

\section{Local Convergence analysis}

We introduce some scalar functions appearing in the local convergence analysis of method (1.2) that follows. Let $\varphi_{0}$ : $\mathbb{R}_{0} \longrightarrow \mathbb{R}_{0}$ be a continuous and non-decreasing function satisfying $\varphi_{0}(0)=0$, where $\mathbb{R}_{0}=\mathbb{R}^{+} \cup\{0\}$. Define the parameter $\rho$ by

$$
\rho=\sup \left\{t \in \mathbb{R}_{0}: \varphi_{0}(t)<1\right\} .
$$

Let $\varphi, \varphi_{1}:[0, \rho) \longrightarrow \mathbb{R}_{0}$ be continuous and nondecreasing functions with $\varphi(0)=\varphi_{1}(0)=0$. Moreover, define functions $g_{1}, g_{2}, g_{3}, h_{1}, h_{2}, h_{3}$ on the interval $[0, \rho)$ by

$$
\begin{gathered}
g_{1}(t)=\frac{\int_{0}^{1} \varphi((1-\theta) t) d \theta}{1-\varphi_{0}(t)}, \\
h_{1}(t)=g_{1}(t)-1, \\
g_{2}(t)=g_{1}(t)+5 \frac{\int_{0}^{1} \varphi_{1}\left(\theta g_{1}(t) t\right) g_{1}(t) d \theta}{1-\varphi_{0}(t)}, \\
h_{2}(t)=g_{2}(t)-1, \\
+\frac{1}{5} \frac{16 \int_{0}^{1} \varphi_{1}\left(\theta g_{1}(t) t\right) g_{1}(t) d \theta+\int_{0}^{1} \varphi_{1}\left(\theta g_{2}(t) t\right) g_{2}(t) d \theta}{1-\varphi_{0}(t)}
\end{gathered}
$$

and

$$
h_{3}(t)=g_{3}(t)-1
$$

Let $\varphi_{2}:[0, \rho)^{2} \longrightarrow \mathbb{R}_{0}$ and $g:[0, \rho) \longrightarrow \mathbb{R}_{0}$ be continuous and nondecreasing functions with $\varphi_{2}(0,0)=0$. Furthermore, define functions $g_{4}$ and $h_{4}$ on the interval $[0, \rho)$ by

$$
g_{4}(t)=g_{3}(t)+g(a) \frac{\int_{0}^{1} \varphi_{1}\left(g_{3}(t) t\right) g_{3}(t) d \theta}{1-\varphi_{0}(t)}
$$

and

$$
h_{4}(t)=g_{4}(t)-1
$$

where

$$
a=\frac{\int_{0}^{1} \varphi_{1}(\theta \rho) \rho d \theta+5 \varphi_{2}\left(g_{1}(\rho) \rho, g_{2}(\rho) \rho\right)}{1-\varphi_{0}(\rho)} .
$$

Using hypothesis (2.1), we obtain $h_{i}(0)=-1<0$ and $h_{i}(t) \longrightarrow+\infty$ as $t \longrightarrow \rho^{-}$for each $i=1,2,3,4$. Then, it follows from the intermediate value theorem that functions $h_{i}$ have zeros in the interval $(0, \rho)$. Denote by $r_{i}$ the smallest zero in $(0, r)$ for each function $h_{i}$, respectively. We have that $h_{i}\left(r_{i-1}\right) \geq 0$, so $r_{4} \leq r_{3} \leq r_{2} \leq r_{1} \leq \rho$. Denote the radius of convergence $r$ by

$$
r=r_{4}=\min \left\{r_{i}\right\}
$$

Then, for each $t \in[0, r)$

$$
0 \leq g_{i}(t)<1
$$

Let $B(u, \lambda):=\left\{x \in \mathcal{B}_{1}:\|x-u\|<\lambda\right\}$ and $\bar{B}(u, \lambda)$ stand for the closure of $B(u, \lambda)$.

The local convergence analysis is based on the conditions $(\mathcal{A})$ :

(a1) $F: \Omega \subset \mathcal{B}_{1} \longrightarrow \mathcal{B}_{2}$ is a Fréchet differentiable operator.

(a2) There exists $p \in \Omega$ such that $F(p)=0$ and $F^{\prime}(p)^{-1} \in$ $\mathcal{L}\left(\mathcal{B}_{2}, \mathcal{B}_{1}\right)$.

(a3) There exists a continuous and nondecreasing function $\varphi_{0}: \mathbb{R}_{0} \longrightarrow \mathbb{R}_{0}$ with $\varphi_{0}(0)=0$ such that for each $x \in \Omega:\left\|F^{\prime}(p)^{-1}\left(F^{\prime}(x)-F^{\prime}(p)\right)\right\| \leq \varphi_{0}(\|x-p\|)$. Set $\Omega_{0}=\Omega \cap B(p, \rho)$, where $\rho$ is defined by (2.1).

(a4)There exists a divided difference $[., . ; F]: \Omega_{0}^{2} \longrightarrow$ $\mathcal{L}\left(\mathcal{B}_{1}, \mathcal{B}_{1}\right)$ satisfying $[x, y ; F](x-y)=F(x)-F(y)$ for each $x, y \in \Omega_{0}$ with $x \neq y$ and $F^{\prime}(x)=[x, x ; F]$.

(a5) There exists continuous and nondecreasing functions $\varphi, \varphi_{1}:[0, \rho) \longrightarrow \mathbb{R}_{0}, \varphi_{2}:[0, \rho)^{2} \longrightarrow \mathbb{R}_{0}$ with $\varphi(0)=$ $\varphi_{1}(0)=0$ such that for each $x, y \in \Omega_{0}$ :

$$
\begin{gathered}
\left\|F^{\prime}(p)^{-1}\left(F^{\prime}(x)-F^{\prime}(y)\right)\right\| \leq \varphi(\|x-y\|), \\
\left\|F^{\prime}(p)^{-1} F^{\prime}(x)\right\| \leq \varphi_{1}(\|x-p\|)
\end{gathered}
$$

and

$$
\left\|F^{\prime}(p)^{-1}[x, y ; F]\right\| \leq \varphi_{2}(\|x-p\|,\|y-p\|)
$$

(a6) $G(Q): \mathcal{B}_{1} \longrightarrow \mathcal{B}_{1}$ is a linear operator for each linear operator $Q: \mathcal{B}_{1} \longrightarrow \mathcal{B}_{1}$ and there exists continuous 
and nondecreasing function $g:[0, \rho) \longrightarrow \mathbb{R}_{0}$ such that $\|G(Q)\| \leq g(\|Q\|)$ where $Q=I-5 F^{\prime}(x)^{-1}[y, z ; F]$, for each $x, y, z \in \Omega_{0}$.

and

(a7) $B(p, r) \subseteq \Omega$, where the radius $r$ is defined by (2.3)

Next, we provide the local convergence analysis of method (1.2) using the conditions $(\mathcal{A})$ and the previously introduced notation.

Theorem 2.1. Suppose that the condition $(\mathcal{A})$ hold. Then, for $x_{0} \in B(p, r)-\{p\}$ the sequence generated by method (1.2) is well defined in $B(p, r)$, remains in $B(p, r)$ for each $n=0,1,2, \ldots$ and converges to $p$. Moreover, the following error bounds hold

$$
\begin{aligned}
\left\|y_{n}-p\right\| & \leq g_{1}\left(\left\|x_{n}-p\right\|\right)\left\|x_{n}-p\right\| \leq\left\|x_{n}-p\right\|<r, \\
\left\|z_{n}-p\right\| \leq g_{2}\left(x_{n}-p \|\right)\left\|x_{n}-p\right\| \leq\left\|x_{n}-p\right\|, & \left\|w_{n}-p\right\| \leq g_{3}\left(\left\|x_{n}-p\right\|\left\|x_{n}-p\right\| \leq\left\|x_{n}-p\right\|\right.
\end{aligned}
$$

and

$$
\left\|x_{n+1}-p\right\| \leq g_{3}\left(\left\|x_{n}-p\right\|\right)\left\|x_{n}-p\right\| \leq\left\|x_{n}-p\right\|,
$$

where the functions $g_{i}$ are defined previously. Furthermore, if there exists $r^{\star} \geq r$ such that

$$
\int_{0}^{1} \varphi_{0}\left(\theta r^{\star}\right) d \theta<1,
$$

then, $p$ is the only solution of equation $F(x)=0$ in $\Omega_{1}=$ $\Omega \cap \bar{B}\left(p, r^{\star}\right)$.

Proof. Sequence $\left\{x_{n}\right\}$ shall be shown to be convergent to $p$ so that error bounds (2.5)-(2.8) hold using mathematical induction. Let $x \in B(p, r)$. Then, using (2.1) (a2) and (a3), we have in turn that

$$
\left\|F^{\prime}(p)^{-1}\left(F^{\prime}(x)-F^{\prime}(p)\right)\right\| \leq \varphi_{0}(\|x-p\|) \leq \varphi_{0}(r) \leq \varphi(\rho)<1 .
$$

The Banach lemma on invertible operators $[4,9,22,24]$ and (2.10) assert that $F^{\prime}(x)^{-1} \in \mathcal{L}\left(\mathcal{B}_{2} \cdot \mathcal{B}_{1}\right)$ and

$$
\left\|F^{\prime}(x)^{-1} F^{\prime}(p)\right\| \leq \frac{1}{1-\varphi_{0}(\|x-p\|)} .
$$

In particular, (2.11) holds for $x=x_{0}$, since $x_{0} \in B(p, r)$, and $y_{0}, z_{0}, w_{0}$ and $x_{1}$ are well defined by method (1.2). We can have by (2.3), (2.4) (for $i=1)$ and (a5) that

$$
\begin{aligned}
\left\|y_{0}-p\right\| \leq & \left\|F^{\prime}\left(x_{0}\right)^{-1} F^{\prime}(p)\right\| \\
& \int_{0}^{1} F^{\prime}(p)^{-1}\left(F^{\prime}\left(p+\theta\left(x_{0}-p\right)\right)-F^{\prime}\left(x_{0}\right)\right)\left(x_{0}-p\right) d \theta \\
\leq & \frac{\int_{0}^{1} \varphi\left((1-\theta)\left\|x_{0}-p\right\|\right) d \theta\left\|x_{0}-p\right\|}{1-\varphi_{0}\left(\left\|x_{0}-p\right\|\right)} \\
= & g_{1}\left(\left\|x_{0}-p\right\|\right)\left\|x_{0}-p\right\| \leq\left\|x_{0}-p\right\|<r
\end{aligned}
$$

which shows (2.5) for $n=0$ and $y_{0} \in B(p, r)$. In view of (a1), (a2) and (a5) we get

$$
F\left(y_{0}\right)=F\left(y_{0}\right)-F(p)=\int_{0}^{1} F^{\prime}\left(p+\theta\left(y_{0}-p\right)\right) d \theta\left(y_{0}-p\right)
$$

so

$$
\begin{aligned}
& \left\|F^{\prime}(p)^{-1} F\left(y_{0}\right)\right\| \\
\leq & \left\|F^{\prime}(p)^{-1} \int_{0}^{1} F^{\prime}\left(p+\theta\left(y_{0}-p\right)\right) d \theta\left(y_{0}-p\right)\right\| \\
\leq & \varphi_{1}\left(\left\|y_{0}-p\right\|\right)\left\|y_{0}-p\right\| \\
\leq & \varphi_{1}\left(g_{1}\left(\left\|x_{0}-p\right\|\right)\left\|x_{0}-p\right\|\right) g_{1}\left(\left\|x_{0}-p\right\|\right)\left\|x_{0}-p\right\|,
\end{aligned}
$$

where we also used that $p+\theta\left(y_{0}-p\right) \in B(p, r)$, since $\| p+$ $\theta\left(y_{0}-p\right)-p\|=\theta\| y_{0}-p \|<r$ for each $\theta \in[0,1]$. Then, by (2.3), (2.4) (for $i=2,3$ ), (2.11) and (2.14), we get in turn that

$$
\begin{aligned}
& \left\|z_{0}-p\right\| \leq\left\|y_{0}-p\right\|+5\left\|F^{\prime}\left(x_{0}\right)^{-1} F^{\prime}(p)\right\|\left\|F^{\prime}(p)^{-1} F\left(y_{0}\right)\right\| \\
& \leq g_{1}\left(\left\|x_{0}-p\right\|\right)\left\|x_{0}-p\right\| \\
& +5 \frac{\int_{0}^{1} \varphi_{1}\left(\theta\left\|y_{0}-p\right\|\right) d \theta\left\|y_{0}-p\right\|}{1-\varphi_{0}\left(\left\|x_{0}-p\right\|\right)} \\
& \leq g_{1}\left(\left\|x_{0}-p\right\|\right)\left\|x_{0}-p\right\| \\
& +5 \frac{\left(\int_{0}^{1} \varphi_{1}\left(\theta g_{1}\left(\left\|x_{0}-p\right\|\right)\left\|x_{0}-p\right\|\right) d \theta\right) g_{1}\left(\left\|x_{0}-p\right\|\right)\left\|x_{0}-p\right\|}{1-\varphi_{0}\left(\left\|x_{0}-p\right\|\right)} \\
& =g_{2}\left(\left\|x_{0}-p\right\|\right)\left\|x_{0}-p\right\| \leq\left\|x_{0}-p\right\|<r
\end{aligned}
$$

and

$$
\begin{aligned}
& \left\|w_{0}-p\right\| \leq\left\|z_{0}-p\right\| \\
& +\frac{1}{5}\left\|F^{\prime}\left(x_{0}\right)^{-1} F^{\prime}(p)\right\|\left[16\left\|F^{\prime}(p)^{-1} F\left(y_{0}\right)\right\|+\left\|F^{\prime}(p)^{-1} F\left(z_{0}\right)\right\|\right] \\
& \leq g_{2}\left(\left\|x_{0}-p\right\|\right)\left\|x_{0}-p\right\| \\
& +\frac{1}{5} \frac{16 \int_{0}^{1} \varphi_{1}\left(\theta\left\|y_{0}-p\right\|\right) d \theta\left\|y_{0}-p\right\|+\int_{0}^{1} \varphi_{1}\left(\theta\left\|z_{0}-p\right\|\right) d \theta\left\|z_{0}-p\right\|}{1-\varphi_{0}\left(\left\|x_{0}-p\right\|\right)} \\
& \leq g_{3}\left(\left\|x_{0}-p\right\|\right)\left\|x_{0}-p\right\| \leq\left\|x_{0}-p\right\|<r,
\end{aligned}
$$

which shows (2.6), (2.7) for $n=0$, respectively and $z_{0}, w_{0} \in$ $B(p, r)$. We need an upper bound on $\left\|s_{0}\right\|$. Notice by (a5), 
(a6), we have that

$$
\begin{aligned}
& \left\|s_{0}\right\| \leq\left\|F^{\prime}\left(x_{0}\right)^{-1}\left(F^{\prime}\left(x_{0}\right)-5\left[y_{0}, x_{0} ; F\right]\right)\right\| \\
& \leq\left\|F^{\prime}\left(x_{0}\right)^{-1} F^{\prime}(p)\right\|\left[\left\|F^{\prime}(p)^{-1} F^{\prime}\left(x_{0}\right)\right\|+5\left\|F^{\prime}(p)^{-1}\left[y_{0}, z_{0} ; F\right]\right\|\right] \\
& \leq \frac{\int_{0}^{1} \varphi_{1}\left(\theta\left\|x_{0}-p\right\|\right) d \theta\left\|x_{0}-p\right\|+5 \varphi_{2}\left(g_{1}(\rho) \rho, g_{2}(\rho) \rho\right)}{1-\varphi_{0}(\rho)}=a
\end{aligned}
$$

and

$$
\left\|G\left(s_{0}\right)\right\| \leq g\left(\left\|s_{0}\right\|\right) \leq g(a) .
$$

In view of (2.3), (2.4) (for $i=4),(2.11),(2.14)$ (for $w_{0}=x_{0}$ ), (2.17) and (2.18) we obtain in turn that

$$
\begin{aligned}
& \left\|x_{1}-p\right\| \leq\left\|w_{0}-p\right\|+\left\|G\left(s_{0}\right)\right\|\left\|F^{\prime}\left(x_{0}\right)^{-1} F^{\prime}(p)\right\|\left\|F^{\prime}(p)^{-1} F\left(w_{0}\right)\right\| \\
& \leq g_{3}\left(\left\|x_{0}-p\right\|\left\|x_{0}-p\right\|+g(a) \frac{\int_{0}^{1} \varphi_{1}\left(\theta\left\|w_{0}-p\right\|\right) d \theta\left\|w_{0}-p\right\|}{1-\varphi_{0}\left(\left\|x_{0}-p\right\|\right)}\right. \\
& \leq g_{4}\left(\left\|x_{0}-p\right\|\right)\left\|x_{0}-p\right\| \leq\left\|x_{0}-p\right\|<r
\end{aligned}
$$

which shows (2.8) for $n=0$ and $x_{1} \in B(p, r)$. The preceding estimates can be repeated, if we replace $x_{0}, y_{0}, z_{0}, w_{0}, x_{1}$ by $x_{k}, y_{k}, z_{k}, w_{k}, x_{k+1}$. This way we complete the induction for (2.5)-(2.8). Then, by the estimate

$$
\left\|x_{k+1}-p\right\| \leq c\left\|x_{k}-p\right\|<r,
$$

where $c=g_{4}\left(\left\|x_{0}-p\right\|\right) \in[0,1)$, we deduce that $\lim _{k \rightarrow \infty} x_{k}=p$ and $x_{k+1} \in B(p, r)$. To complete the proof, let $p^{\star} \in \Omega_{1}$ be such that $F\left(p^{\star}\right)=0$. Using (a3) and (2.9) for $T=\int_{0}^{1} F^{\prime}\left(p+\theta\left(p^{\star}-p\right)\right) d \theta$, we get that

$$
\begin{aligned}
\left\|F^{\prime}(p)^{-1}\left(T-F^{\prime}(p)\right)\right\| & \leq \int_{0}^{1} \varphi_{0}\left(\theta\left\|p-p^{\star}\right\|\right) d \theta \\
& \leq \int_{0}^{1} \varphi_{0}\left(\theta r^{\star}\right)<1,
\end{aligned}
$$

so $T^{-1} \in \mathcal{L}\left(\mathcal{B}_{2}, \mathcal{B}_{1}\right)$. Finally, from the identity

$$
0=F\left(p^{\star}\right)-F(p)=T\left(p^{\star}-p\right),
$$

we conclude that $p^{\star}=p$.

Remark 2.2. (a) In the case when $\varphi_{0}(t)=L_{0} t, \varphi(t)=L t$ and $\Omega_{0}=\Omega$, the radius $r_{A}=\frac{2}{2 L_{0}+L}$ was obtained by Argyros in [2] as the convergence radius for Newton's method under condition (2.7)-(2.9). Notice that the convergence radius for Newton's method given independently by Rheinboldt [15] and Traub [27] is given by

$$
\rho=\frac{2}{3 L}<r_{A} .
$$

As an example, let us consider the function $H(x)=$ $e^{x}-1$. Then $x^{\star}=0$. Set $\Omega=B(0,1)$. Then, we have that $L_{0}=e-1<L=e$, so $\rho=0.24252961<r_{A}=$ 0.324947231 .

Moreover, the new error bounds [2] are:

$$
\left\|x_{n+1}-x^{\star}\right\| \leq \frac{L}{1-L_{0}\left\|x_{n}-x^{\star}\right\|}\left\|x_{n}-x^{\star}\right\|^{2},
$$

whereas the old ones $[5,7]$

$$
\left\|x_{n+1}-x^{\star}\right\| \leq \frac{L}{1-L\left\|x_{n}-x^{\star}\right\|}\left\|x_{n}-x^{\star}\right\|^{2} .
$$

Clearly, the new error bounds are more precise, if $L_{0}<$ L. Clearly, we do not expect the radius of convergence of method (1.2) given by $r_{3}$ to be larger than $r_{A}$.

(b) The local results can be used for projection methods such as Arnoldi's method, the generalized minimum residual method(GMREM), the generalized conjugate method(GCM) for combined Newton/finite projection methods and in connection to the mesh independence principle in order to develop the cheapest and most efficient mesh refinement strategy [1-5].

(c) The results can be also be used to solve equations where the operator $H^{\prime}$ satisfies the autonomous differential equation [2-4]:

$$
H^{\prime}(x)=P(H(x)),
$$

where $P$ is a known continuous operator. Since $H^{\prime}\left(x^{\star}\right)=$ $P\left(H\left(x^{*}\right)\right)=P(0)$, we can apply the results without actually knowing the solution $x^{\star}$. Let as an example $H(x)=$ $e^{x}-1$. Then, we can choose $P(x)=x+1$ and $x^{\star}=0$.

(d) It is worth noticing that method (1.2) are not changing if we use the new instead of the old conditions [3]. Moreover, for the error bounds in practice we can use the computational order of convergence (COC)

$$
\xi=\frac{\ln \frac{\left\|x_{n+2}-x^{*}\right\|}{\left\|x_{n+1}-x^{*}\right\|}}{\ln \frac{\left\|x_{n+1}-\chi^{*}\right\|}{\left\|x_{n}-x^{*}\right\|}}, \quad \text { for each } n=1,2, \ldots
$$

or the approximate computational order of convergence (ACOC)

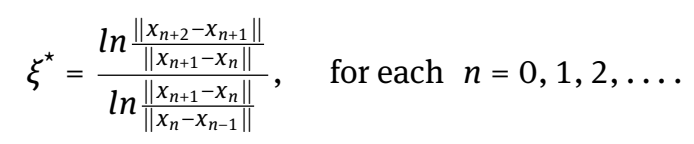

(e) In view of (2.4) and the estimate

$$
\begin{aligned}
\left\|H^{\prime}\left(x^{\star}\right)^{-1} H^{\prime}(x)\right\| & =\left\|H^{\prime}\left(x^{\star}\right)^{-1}\left(H^{\prime}(x)-H^{\prime}\left(x^{\star}\right)\right)+I\right\| \\
& \leq 1+\left\|H^{\prime}\left(x^{\star}\right)^{-1}\left(H^{\prime}(x)-H^{\prime}\left(x^{\star}\right)\right)\right\| \\
& \leq 1+\varphi_{0}\left(\left\|x-x^{\star}\right\|\right)
\end{aligned}
$$

condition (2.6) can be dropped and can be replaced by

$$
v(t)=1+\varphi_{0}(t)
$$

or

$$
v(t)=1+\varphi_{0}(\rho)
$$

since $t \in[0, \rho)$. 


\section{Numerical Examples}

We present two examples in this section using $[x, y ; F]=$ $\int_{0}^{1} F^{\prime}(x+\theta(y-x)) d \theta$ and $Q(s)=I$ in both examples.

Example 3.1. Let $\mathcal{B}_{1}=\mathcal{B}_{2}=\mathbb{R}^{3}, D=\bar{U}(0,1), x^{\star}=$ $(0,0,0)^{T}$. Define function $H$ on $D$ for $w=(x, y, z)^{T}$ by

$$
H(w)=\left(e^{x}-1, \frac{e-1}{2} y^{2}+y, z\right)^{T} .
$$

Then, the Fréchet-derivative is given by

$$
H^{\prime}(v)=\left[\begin{array}{ccc}
e^{x} & 0 & 0 \\
0 & (e-1) y+1 & 0 \\
0 & 0 & 1
\end{array}\right]
$$

Using (2.5)-(2.7), we can choose $\varphi_{0}(t)=L_{0} t, \varphi(t)=$ $e^{\frac{1}{L_{0}}} t, \varphi_{1}(t)=e^{\frac{1}{L_{0}}}, \varphi_{2}(s, t)=e^{\frac{1}{L_{0}}}$ and $g(t)=1$ with $L_{0}=$ $e-1$.

Then, the radius of convergence $r$ is given by

$$
\begin{aligned}
& r=r_{4}=0.0601<r_{2}=0.0836<r_{3}=0.1345 \\
&<r_{1}=0.3827 .
\end{aligned}
$$

Example 3.2. Returning back to the motivational example given at the introduction of this study, we can choose (see also Remark 2.2 (5) for function $v) \varphi_{0}(t)=\varphi(t)=\frac{1}{8}\left(\frac{3}{2} \sqrt{t}+t\right)$ and $\varphi_{1}(t)=1+\varphi_{0}(\rho), \rho \simeq 4.7354, \varphi_{2}(s, t)=1+\varphi_{0}(\rho)$ and $g(t)=1$. Then, the radius of convergence $r$ is given by

$$
\begin{aligned}
& r=r_{4}=0.2130<r_{3}=0.5072<r_{2}=0.9738 \\
&<r_{1}=1.6218 .
\end{aligned}
$$

\section{References}

[1] S. Amat, S. Busquier and M. Negra, Adaptive approximation of nonlinear operators, Numer. Funct. Anal. Optim. 25 (2004) 397-405.

[2] S. Amat, I. K. Argyros, S. Busquier and A. A. Magreñán, Local convergence and the dynamics of a two-point four parameter Jarratt-like method under weak conditions, Numer. Algor., (2017), DOI: 10.1007/s11075-016-0152-5.

[3] A. Cordero, J. M. Gutiérrezb. A. A. Magreñán and J. R.Torregrosa, Stability analysis of a parametric family of iterative methods for solving nonlinear models, Appl. Math. and Comput., 285, 20 (2016), 26-40.

[4] I.K. Argyros, Computational theory of iterative methods. Series: Studies in Computational Mathematics, 15, Editors: C.K.Chui and L. Wuytack, Elsevier Publ. Co. New York, U.S.A, 2007.

[5] I.K. Argyros and H. Ren, Improved local analysis for certain class of iterative methods with cubic convergence, Numerical Algorithms, 59(2012), 505-521.
[6] I.K.Argyros, Yeol Je Cho and S. George, Local convergence for some third-order iterative methods under weak conditions, J. Korean Math. Soc. 53 (2016), No. 4, pp. 781-793.

[7] I.K.Argyros and S. George, Ball convergence of a sixth order iterative method with one parameter for solving equations under weak conditions, Calcolo, 53(4), (2016), pp: 585-595.

[8] I. K. Argyros and A. A. Magreñán , Local convergence analysis of proximal Gauss-Newton method for penalized nonlinear least square problems, Appl. Math. Comput.V. 241, 15(2014), 401-408

[9] I.K.Argyros and F.Szidarovszky, The theory and Applications of Iterative methods,CRC Press,Boca Raton Florida,USA,1993.

[10] A. Cordero, J. Hueso, E. Martinez, J. R. Torregrosa, A modified Newton-Jarratt's composition, Numer. Algor. 55, (2010), 87-99.

[11] A. Cordero, J. Garćia-Maimo, J. R. Torregrosa, M. P. Vassileva and P. Vindel, Chaos in King's iterative family, Appl. Math. Lett., 26 (2013), 842-848.

[12] A. Cordero,E. Gómez and J. R. Torregrosa, Efficient high-order iterative methods for solving nonlinear systems and their application on heat conduction problems, Complexity 2017 (2017), Article ID 6457532, ii pages.

[13] A. Cordero, J. R. Torregrosa, Variants of Newton's method for functions of several variables, Appl.Math. Comput. 183,(2006), 199-208.

[14] A. Cordero, J. R. Torregrosa, Variants of Newton's method using fifth order quadrature formulas, Appl.Math. Comput. 190,(2007), 686-698.

[15] Y. H. Geun, Y. I. Kim and B. Neta, A sixth order family of threepoint modified Newton-like multiple-root finders and the dynamics behind their extraneous fixed points, Appl. Math. Comput. 283(2016), 120-140.

[16] G.M Grau-Sanchez, A.Grau, M .Noguera, On the computational efficiency index and some iterative methods for solving systems of non-linear equations, J. Comput. Appl Math., 236 (2011), 1259-1266.

[17] J. M. Gutiérrez and M. A. Hernández, Newton's method under weak Kantorovich conditions, IMA J. Numer. Anal., 20 (2000), 521-532.

[18] J. M. Gutiérrez, M. A. Hernández and N. Romero, Dynamics of a new family of iterative processes for quadratic polynomials, J. Comput. Appl. Math., 233 (2010), 2688-2695.

[19] A. A. Magreñán, Different anomalies in a Jarratt family of iterative root finding methods, Appl. Math. Comput. 233, (2014), 29-38.

[20] A. A. Magreñán, A new tool to study real dynamics: The convergence plane, Appl. Math. Comput. 248, (2014), 29-38.

[21] M. A. Noor and M. Waseem, Some iterative methods for solving a system of nonlinear equations, Computers and Mathematics with Applications, 57, (2009), 101-106.

[22] A. M. Ostrowski, Solution of the equations and systems of equations, Prentice- Hall, England Cliff, New York, 1964.

[23] A.N .Romero, J.A. Ezquerro, M .A. Hernandez, Approximacion de soluciones de algunas equacuaciones integrals de Hammerstein mediante metodos iterativos tipo. Newton, XXI Congresode ecuaciones diferenciales y aplicaciones Universidad de Castilla-La Mancha (2009)

[24] W.C. Rheinboldt,An adaptive continuation process for solving systems of nonlinear equations, In: Mathematical models and numerical methods (A.N.Tikhonov et al. eds.) pub.3, (1977), 129-142 Banach Center, Warsaw Poland. 
[25] J. R. Sharma and H. Arora, Improved Newton like methods for solving systems of nonlinear equations, SeMA Journal, 74(2), (2017), 147-163.
[26] J.R. Sharma, P.K. Gupta, An efficient fifth order method for solving systems of nonlinear equations, Comput. Math. Appl. 67, (2014), 591-601.

[27] J.F.Traub, Iterative methods for the solution of equations, AMS Chelsea Publishing, 1982. 